\title{
Progressive Multifocal Leukoencephalopathy after Ibrutinib Therapy for Chronic Lymphocytic Leukemia
}

\author{
Mathias Lutz, MD \\ Arik B. Schulze, MD \\ Elisabeth Rebber, MD',2 \\ Stefanie Wiebe, MD \\ Tarek Zoubi, MD ${ }^{3}$ \\ Oliver M. Grauer, MD, PhD ${ }^{4}$ \\ Torsten Keßler, MD' \\ Andrea Kerkhoff, MD 1 \\ Georg Lenz, MD $1,5,6$ \\ Wolfgang E. Berdel, MD1
}

${ }^{1}$ Department of Medicine A,

University Hospital of Münster, Münster, ${ }^{2}$ Department of Internal Medicine, Marienhospital Osnabrück, Osnabrück, Departments of ${ }^{3}$ Clinical Radiology, ${ }^{4}$ Neurology, and ${ }^{5}$ Translational Oncology,

University Hospital of Münster, Münster, ${ }^{6}$ Cluster of Excellence EXC 1003,

Cells in Motion, Münster, Germany

Correspondence: Mathias Lutz, MD

Department of Medicine A, University Hospital of Münster, Albert-Schweitzer-Campus 1,

D-48149 Münster, Germany

Tel: 49-251-83-47587

Fax: 49-251-83-47588

E-mail: mathias.lutz@ukmuenster.de

Received March 15, 2016

Accepted June 15, 2016

Published Online July 12, 2016
Progressive multifocal leukoencephalopathy $(\mathrm{PML})$ is a devastating neurological disease observed nearly exclusively in immunocompromised patients. Recently, the introduction of monoclonal antibodies significantly inhibiting the immune system such as rituximab has led to an increase in PML cases. Although rituximab-based immunochemotherapy remains the standard of treatment for chronic lymphocytic leukemia (CLL), the importance of Bruton's tyrosine kinase inhibitors such as ibrutinib is steadily increasing. However, long-term experiences regarding possible side effects of these new substances are rare. Here, we report the development of eventually fatal PML possibly associated with ibrutinib therapy for CLL after multiple prior treatment lines, including rituximab. To the best of our knowledge, this is the first study to report such findings. Since the last course of rituximab was applied over 3 years ago, it is conceivable that the strong $B$ cell inhibition by ibrutinib led to PML. With increased awareness of this potential side effect, further clinical studies are certainly warranted to evaluate this possible association.

\section{Introduction}

Progressive multifocal leukoencephalopathy (PML) is a rare and often fatal demyelinating disease of the central nervous system (CNS) that is nearly exclusively seen in immunocompromised patients. PML is caused by reactivation of JC virus (JCV), which subsequently invades oligodendrocytes and astrocytes, thereby leading to their irreparable degener-

\section{Key words}

B-Cell chronic lymphocytic leukemia, Ibrutinib, Rituximab, Progressive multifocal leukoencephalopathy, JC virus 
malignancies and/or treatment with immunosuppressive drugs. The epidemiology of PML was dramatically changed by the emergence of acquired immune deficiency syndrome (AIDS). Indeed, up to $5 \%$ of all AIDS patients developed the disease, accounting for about $80 \%$ of all PML cases. In the following decades, the introduction of highly active antiretroviral therapy regimens led to a remarkable decrease in its incidence as well as mortality [1,4].

However, PML moved back into focus after introduction of therapeutic approaches based on monoclonal antibodies. Soon after its introduction in 2004, first cases of patients developing PML after treatment with the alpha-4 integrin antibody natalizumab for multiple sclerosis or Crohn disease were reported $[2,4]$. To date, the overall PML risk for patients on natalizumab was estimated to be 4 to 11 per 1,000 depending on anti-JCV antibody status, treatment duration and prior history of immunosuppression [5]. In the following years, other monoclonal antibodies have also been suspected to increase the incidence of PML. Among these, the CD20 antibody rituximab is playing an important role because of its widespread use in different B-cell lymphomas, and a remarkable number of PML cases have been reported after rituximab treatment $[4,6]$.

Although rituximab-based immunochemotherapy remains the standard of first-line treatment for B-cell lymphomas such as CLL, the importance of small molecules is steadily increasing, especially in patients with refractory or relapsed disease and in those whose initial therapeutic approach is limited by toxicities. One group of these small molecules consists of inhibitors of Bruton's tyrosine kinase (BTK), a key enzyme in the signaling pathway downstream of the B cell receptor (BCR). Following antigen-dependent triggering of the BCR, BTK is activated, subsequently leading to inhibition of B-cell apoptosis and promotion of B-cell proliferation [7].

Ibrutinib, formerly known as PCI-32765, is an orally bioavailable selective BTK inhibitor that is administered once-daily to prevent B-cell differentiation, proliferation, and survival [7]. In different studies, ibrutinib was highly active in different B-cell lymphomas such as CLL or mantle cell lymphoma combined with a modest toxicity profile [8]. However, few studies have investigated the possible longterm side effects of ibrutinib.

Here, we report the first case of a patient with CLL who developed PML after receiving ibrutinib therapy. To the best of our knowledge, this is the first study to report such findings.

\section{Case Report}

\section{Initial diagnosis and treatment of CLL}

The reported human immunodeficiency virus-negative male patient initially presented in 2005 at the age of 65 years with moderate lymphadenopathy of the cervical, axillary, abdominal, and inguinal lymph nodes, with no hepatomegaly or splenomegaly. The hemogram revealed lymphocytosis without anemia or thrombocytopenia (Binet stage B). A consecutively conducted bone marrow examination revealed $25 \%$ infiltration by CLL of B-cell origin without CD20 expression. After observation for over 3 years, the patient showed a general disease progression in November of 2008. At that time, bone marrow examination showed $70 \%$ CLL infiltration of the marrow with partially strong and partially weak CD20 expression. Subsequently, therapy with a total of five cycles fludarabine and cyclophosphamide was initiated in private practice. According to the International Workshop Group on CLL (IWCLL) response criteria, complete remission with incomplete bone marrow recovery (CRi) was achieved after three cycles of this treatment regimen.

\section{Rituximab-based and further treatment}

In February 2012, a significant progression of lymph node manifestations was observed and immunochemotherapy with rituximab and bendamustine was initiated. Two months later, this treatment had to be discontinued because of a newly diagnosed mixed axonal and demyelinating sensorimotor polyneuropathy resulting in hypoesthesia of the trunk, paresthesia of the fingers, slight tetraparesis, mild ptosis, and mild dysarthria. At that time, CRi was achieved. Disease stability was observed during the following months until a recurrent progression in February 2013. Subsequently, a subcutaneous venous port catheter was surgically implanted and the patient was treated with six cycles of cyclophosphamide, epirubicin, and prednisolone. After a phase of stable disease parameters, CLL progressed again in September 2013 with signs of Coombs-positive hemolysis. Three consecutively administered cycles of fludarabine monotherapy had to be substituted by cyclophosphamide because of the insufficient initial response to treatment. However, this regimen had to be discontinued after three cycles because of a beginning bone marrow failure. At that time, a stable disease (according to IWCLL response criteria) was achieved and persisted for more than 1 year. 


\section{Treatment with ibrutinib}

In March 2015, a new disease progression manifesting with lymphadenopathy and hemolysis was treated by initiation of monotherapy with ibrutinib (420 mg daily), which was initially well tolerated by the patient. However, he had to be admitted to our hospital 3 weeks later with fever caused by bilateral pneumonia; therefore, ibrutinib therapy was suspended after a total treatment period of 27 days. The initial antibiotic regimen consisted of piperacillin-tazobactam and clarithromycin and was supplemented by therapeutic doses of co-trimoxazole because of suspected Pneumocystis pneumonia. A consecutively performed bronchoalveolar lavage was positive for Escherichia coli, Candida albicans, Candida krusei, and herpes simplex virus. Hence, the therapeutic regimen was shifted to ceftriaxone, caspofungin, and therapeutic doses of acyclovir, resulting in rapid cessation of fever. During hospital stay, chlorambucil was administered intermittently to reduce leukocyte counts and ibrutinib therapy was not resumed again. After recovery, the patient was discharged to a nursing facility for one week and was able to return home afterwards.

\section{Diagnosis and treatment of PML}

In May of 2015, the patient was again admitted to our hospital with fever and radiographic evidence of interstitial pneumonia. Because of sustained fever, initial empiric antibiotic therapy with piperacillin-tazobactam and clar- ithromycin was replaced by meropenem and vancomycin and intravenous immunoglobulins were administered to substitute for a lack of immunoglobulins (IgG, $387 \mathrm{mg} / \mathrm{dL}$ ). Furthermore, the prophylactic antimycotic regimen was replaced by caspofungin according to resistogram after detection of Saccharomyces cerevisiae in the sputum. Subsequently, fever resolved rapidly and regression of pulmonary infiltration was demonstrated by chest radiography over the course of the next few days.

However, additional newly diagnosed and rapidly progressive neurological symptoms consisting of confusion, gait disorder and urinary incontinence were noted during the patient's hospital stay. An initial cranial computed tomography $(\mathrm{CT})$ scan demonstrated hypodense lesions in the right frontal lobe that were interpreted to be of cicatricial nature. A consecutively conducted lumbar puncture revealed normal cytological and neurochemical parameters of cerebrospinal fluid (CSF) and no increased intracranial pressure $\left(12 \mathrm{cmH}_{2} \mathrm{O}\right)$. Two days later, cranial magnetic resonance (MR) imaging showed the known lesions in the right frontal lobe as hypointense in T1-weighted images and hyperintense in T2-weighted and fluid-attenuated inversion recovery (FLAIR) images without mass effect and with no enhancement of contrast media (Fig. 1). Subsequently, a second lumbar puncture performed because of strong suspicion of PML revealed a highly positive JCV DNA $\left(1.5 \times 10^{7}\right.$ copies $\left./ \mathrm{mL}\right)$. All other cytological, neurochemical, microbiological and virological tests were normal and PML was diagnosed.

After notification of the diagnosis and prognosis of PML,
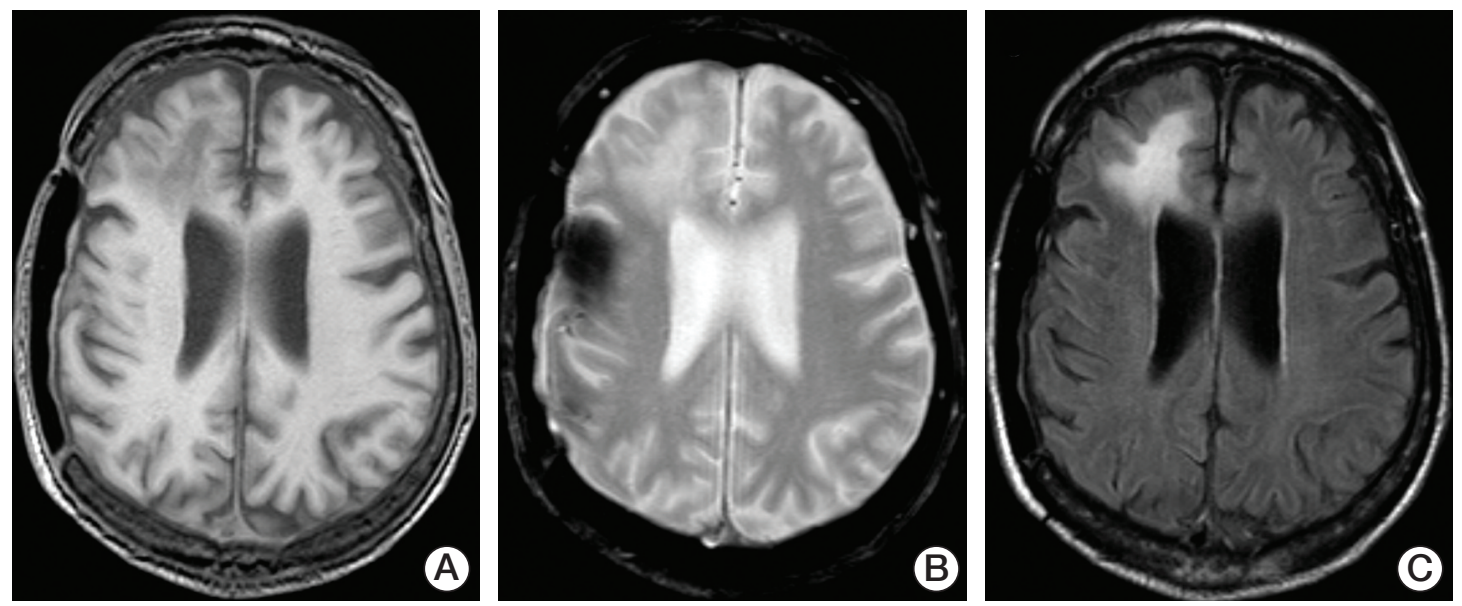

Fig. 1. Cranial magnetic resonance imaging demonstrated asymmetric lesions without mass effect or enhancement of contrast media predominantly located in the right frontal lobe. In 2004, decompressive craniectomy was performed in the patient to treat intracerebral hemorrhage. Sequelae of this neurosurgical procedure can be seen on the right temporoparietal side. The same sagittal section of the brain is shown as T1-weighted (A), T2-weighted (B), and fluid-attenuated inversion recovery (C) images. 
the patient was discharged on his own and his family's request with a combined oral therapy regimen with mefloquine (250 $\mathrm{mg}$ daily for three days followed by $250 \mathrm{mg}$ once weekly) and mirtazapine (60 mg daily) as proposed in the literature [9]. However, neurological symptoms progressed continuously over the following weeks and the patient died seven weeks after diagnosis of PML at the age of 75 years in a nursing home, most likely due to aspiration pneumonia.

\section{Discussion}

Here, we report the eventually fatal case of a 75-year-old male patient with CLL who was pretreated with rituximab and developed PML after receiving monotherapy with ibrutinib, a novel BTK inhibitor. The patient initially presented with typical clinical signs of PML, including speech disorders, cognitive impairment and motor symptoms evolving over days to weeks. Precise neurological symptoms experienced by patients depend on the site of cerebral lesions caused by PML. Therefore, mild gait disorders are likely, as are epileptic seizures [1].

Initial suspicion of PML is often established by cranial imaging performed after appearance of the first neurological symptoms. When compared to CT, MR imaging demonstrates greater sensitivity for visualization of single lesions of PML in the brain and is therefore considered the technique of choice [2]. Cerebral lesions are typically located in both hemispheres in an asymmetric manner, preferably involving subcortical and periventricular white matter in the frontal or parietooccipital lobes. However, involvement of cortical areas has also been reported. Single lesions vary in size and shape, generally becoming larger and more confluent during PML progression. While PML lesions appear hypoattenuating on CT scans, MR imaging shows them as hypointense in T1-weighted images and hyperintense in T2-weighted and FLAIR images. Usually, no mass effect and no enhancement of contrast media is observed. Since signal changes in T1- and T2-weighted MR images are irreversible in most PML cases, diffusion-weighted MR imaging (DW-MRI) constitutes a useful tool to monitor the course of PML. Cytotoxic edema associated with disease progression results in DW-MRI hyperintensity, while quiescent disease areas lead to low signals on DW-MRI [1,2]. Although brain biopsy remains the gold standard to definitively diagnose PML, most cases are diagnosed via polymerase chain reaction (PCR)-based detection of JCV DNA in the CSF. This was also the case in our patient, who was found to have $1.5 \times 10^{7}$ copies $/ \mathrm{mL}$. However, it is important to note that cases with negative JCV PCR results in CSF samples have been reported, despite biopsy-confirmed diagnosis of PML [4].

At present, there is no specific agent to treat PML in a satisfactory manner. To date, no approaches to introduce antiviral drugs to the treatment of PML have demonstrated reasonable efficacy. While cytarabine resulted in decreased replication and multiplication of JCV in a cell culture system, its intravenous or intrathecal application in AIDS patients with PML failed to demonstrate survival benefits in a clinical trial [10]. Different case reports described a possible efficacy of cidofovir, but a prospective pilot study of cidofovir in AIDS patients with PML showed no benefit in MR imaging abnormalities and neurological examination scores [11]. Since the JCV invasion of oligodendrocytes is potentially mediated by binding to the serotonergic 5 -HT2 $\alpha$ receptor, serotonin receptor antagonists such as the antidepressant mirtazapine or the atypical antipsychotic risperidone have been proposed to block JCV from entering glial cells. However, the results of various case reports are inconsistent, and evidence of efficacy remains lacking [9]. The employment of mefloquine, an antimalarial drug, has been proposed for treatment in response to an in vitro infection assay screening about 2,000 agents for their activity against JCV because it was effective against JCV and showed sufficient penetration into the CNS [12]. A small number of case reports showed mitigated PML courses under mefloquine treatment [9]. However, a randomized proof-of-concept study comparing patients receiving standard care to those receiving additional mefloquine therapy failed to show significant differences in clinical or MR imaging results as well as in JCV DNA loads in the CSF [12]. In our case, a combined regimen of mirtazapine ( $60 \mathrm{mg}$ daily) and mefloquine ( $250 \mathrm{mg}$ daily for 3 days followed by $250 \mathrm{mg}$ once weekly) was initiated after initial diagnosis of PML, but was not effective to stop disease progression, leading to fatal outcome seven weeks after diagnosis. Recently, employment of maraviroc, a small molecule inhibitor of chemokine receptor 5, has been discussed to block JCV entry and spread [13]. However, only a small number of cases have been reported and further clinical studies are warranted. To date, reconstitution of the patient's immune system remains the only option confirmed to successfully treat development of PML [1,2].

Although individual patients with late onset of PML after rituximab treatment have been reported [14], most cases described in the literature occurred within 6 to 12 months after the last application of rituximab [6]. Since our patient received his last rituximab application over 36 months prior to diagnosis of PML, it seems unlikely that rituximab was the only cause of PML development in this case. The same is true for fludarabine. Development of PML after fludarabine treatment have been described in a limited number of case reports, and nearly always in combination with rituximab [15]. Additionally, fludarabine was discontinued about 15 
months before PML diagnosis in our case. Therefore, treatment with the novel BTK inhibitor ibrutinib introduced several weeks before the appearance of first clinical PML symptoms and lasting for nearly 1 month must be taken into account. Inhibition of BTK-dependent pathways leads to strong repression of B-cell proliferation and survival [8]. As B-cell-mediated immunity is known to play an important role in control of JCV replication via humoral immune responses, as well as its impact on T-cell activity [1,4], it is conceivable that the introduction of ibrutinib could have triggered JCV reactivation in this case.

This report is intended to increase awareness of this potential side effect. Therefore, risk stratification for development of PML via evaluation of JCV antibody status should be recommended prior to introduction of treatment with ibrutinib. However, further studies are warranted to evaluate the possible association of ibrutinib therapy with development of PML.

\section{Conflicts of Interest}

Conflict of interest relevant to this article was not reported.

\section{References}

1. Ferenczy MW, Marshall LJ, Nelson CD, Atwood WJ, Nath A, Khalili K, et al. Molecular biology, epidemiology, and pathogenesis of progressive multifocal leukoencephalopathy, the JC virus-induced demyelinating disease of the human brain. Clin Microbiol Rev. 2012;25:471-506.

2. Honce JM, Nagae L, Nyberg E. Neuroimaging of natalizumab complications in multiple sclerosis: PML and other associated entities. Mult Scler Int. 2015;2015:809252.

3. Astrom KE, Mancall EL, Richardson EP Jr. Progressive multifocal leuko-encephalopathy: a hitherto unrecognized complication of chronic lymphatic leukaemia and Hodgkin's disease. Brain. 1958;81:93-111.

4. Tavazzi E, Ferrante P, Khalili K. Progressive multifocal leukoencephalopathy: an unexpected complication of modern therapeutic monoclonal antibody therapies. Clin Microbiol Infect. 2011;17:1776-80.

5. Bloomgren G, Richman S, Hotermans C, Subramanyam M, Goelz S, Natarajan A, et al. Risk of natalizumab-associated progressive multifocal leukoencephalopathy. N Engl J Med. 2012;366:1870-80.

6. Carson KR, Evens AM, Richey EA, Habermann TM, Focosi D, Seymour JF, et al. Progressive multifocal leukoencephalopathy after rituximab therapy in HIV-negative patients: a report of 57 cases from the Research on Adverse Drug Events and Reports project. Blood. 2009;113:4834-40.

7. Novero A, Ravella PM, Chen Y, Dous G, Liu D. Ibrutinib for B cell malignancies. Exp Hematol Oncol. 2014;3:4.

8. Tucker DL, Rule SA. A critical appraisal of ibrutinib in the treatment of mantle cell lymphoma and chronic lympho- cytic leukemia. Ther Clin Risk Manag. 2015;11:979-90.

9. Epperla N, Medina-Flores R, Mazza JJ, Yale SH. Mirtazapine and mefloquine therapy for non-AIDS-related progressive multifocal leukoencephalopathy. WMJ. 2014;113:242-5.

10. Hall CD, Dafni U, Simpson D, Clifford D, Wetherill PE, Cohen $\mathrm{B}$, et al. Failure of cytarabine in progressive multifocal leukoencephalopathy associated with human immunodeficiency virus infection. AIDS Clinical Trials Group 243 Team. N Engl J Med. 1998;338:1345-51.

11. Marra CM, Rajicic N, Barker DE, Cohen BA, Clifford D, Donovan Post MJ, et al. A pilot study of cidofovir for progressive multifocal leukoencephalopathy in AIDS. AIDS. 2002;16: 1791-7.

12. Clifford DB, Nath A, Cinque P, Brew BJ, Zivadinov R, Gorelik $\mathrm{L}$, et al. A study of mefloquine treatment for progressive multifocal leukoencephalopathy: results and exploration of predictors of PML outcomes. J Neurovirol. 2013;19:351-8.

13. Giacomini PS, Rozenberg A, Metz I, Araujo D, Arbour N, BarOr A, et al. Maraviroc and JC virus-associated immune reconstitution inflammatory syndrome. N Engl J Med. 2014;370: 486-8.

14. Lane MA, Renga V, Pachner AR, Cohen JA. Late occurrence of PML in a patient treated for lymphoma with immunomodulatory chemotherapies, bendamustine, rituximab, and ibritumomab tiuxetan. Case Rep Neurol Med. 2015;2015:892047.

15. Lejniece S, Murovska M, Chapenko S, Breiksa B, Jaunmuktane $\mathrm{Z}$, Feldmane L, et al. Progressive multifocal leukoencephalopathy following fludarabine treatment in a chronic lymphocytic leukemia patient. Exp Oncol. 2011;33:239-41. 